\title{
REgENERACIÓN DE BULBOS DE HIPPEASTRUM STRIATUM Y HABRANTHUS BRACHYANDRUS (AMARYLLIDACEAE) SOMETIDOS A CORTE LONGITUDINAL
}

\author{
ORLANDO A. RODRIGUEZ MATA ${ }^{1}$, ANA I. HONFI ${ }^{1, *}$ y JULIO R. DAVIÑA ${ }^{1}$
}

\begin{abstract}
Summary: Regeneration of bulbs of Hippeastrum striatum and Habranthus brachyandrus (Amaryllidaceae) subjected to longitudinal cutting. As an alternative for increasing the vegetative propagation of two ornamental promising species, Hippeastrum striatum and Habranthus brachyandrus, the capacity of vegetative regeneration of bulbs subjected to cut, was analyzed. For both species the number of bulblets was greater when the mother bulb was cut. In H. striatum 13 - 20 regenerated bulblets were obtained with diameters between 1.02 and $1.16 \mathrm{~cm}(x=1.09 \mathrm{~cm})$. In $H$. brachyandrus $16-23$ new bulblets were obtained with diameters between 0.87 and $0.94 \mathrm{~cm}(x=0.91 \mathrm{~cm})$. The number of leaves in new bulblets varied between 5 to 0 leaves/bulb in $H$. striatum and between 3 to 0 in $H$. brachyandrus. For bulbs that were not subjected to cutting (control treatment), there was no production of bulblets in $H$. brachyandrus, instead, in $H$. striatum smaller size bulblets were obtained without signs of leaves development. The mechanical induction of bulblet development from mother bulbs, had a highly significant effect in the variables evaluated, stimulating the activation of basal scale buds giving rise to an asexual progeny of clonal origin.
\end{abstract}

Key words: Amaryllidaceae, Asexual progeny, bulblets, vegetative propagation.

Resumen: La capacidad de regeneración vegetativa de bulbos de Hippeastrum striatum y Habranthus brachyandrus sometidos a corte longitudinal, fue analizada como alternativa para la propagación vegetativa de estas especies ornamentales promisorias. En ambas, el número de bulbillos hijos producido fue mayor cuando el bulbo madre fue cortado. En $\mathrm{H}$. striatum se regeneraron entre 13 y 20 bulbillos con diámetros que variaron entre los $1,02 \mathrm{~cm}$ y $1,16 \mathrm{~cm}(x=1,09 \mathrm{~cm})$. En $H$. brachyandrus se obtuvieron de 16 a 23 bulbillos con diámetros que variaron entre los $0,87 \mathrm{~cm}$ y $0,94 \mathrm{~cm}(x=0,91 \mathrm{~cm})$. La cantidad de hojas en los bulbillos regenerados varió entre 5 y 0 hojas/bulbillo en $H$. striatum y entre 3 y 0 en $H$. brachyandrus. Los bulbos que no fueron sometidos a corte (tratamiento control), no produjeron bulbillos en $H$. brachyandrus, en cambio en $H$. striatum se obtuvieron bulbillos de menor tamaño sin indicios de desarrollo de hojas. La inducción mecánica a través de corte longitudinal en los bulbos tuvo un efecto altamente significativo en las variables evaluadas, estimulando el proceso de activación de las yemas basales de las escamas originando la progenie asexual de origen clonal.

Palabras clave: Amaryllidaceae, bulbillos, Progenie asexual, propagación vegetativa.

\section{INTRODUCCIÓN}

La reproducción asexual en plantas y el origen de progenie genéticamente idéntica a la madre, ocurre con diferentes modalidades, como apomixis (agamospermia), embrionía adventicia, y multiplicación o propagación vegetativa a través del desarrollo de estolones, caules subterráneos o rizomas, bulbos, tubérculos, yemas foliares, entre otros (Hartmann \& Kester, 1975; De Abate, 1999; Batygina, 2009). Los bulbos son conocidos como caules especializados para el almacenamiento de reservas y poseen yemas con capacidad de formación de bulbos hijos, que resultan clones de

\footnotetext{
1 Programa de Estudios Florísticos y Genética Vegetal, Instituto de Biología Subtropical (CONICET-UNaM), nodo Posadas, Facultad de Ciencias Exactas, Químicas y Naturales. Rivadavia 2370, 3300 Posadas, Misiones.

*ahonfi@gmail.com
} 


\section{Bol. Soc. Argent. Bot. 53 (4) 2018}

la planta madre (Raven et al., 2007; Batygina, 2009; Baranova, 2009). La presencia de bulbos no es muy frecuente en la naturaleza, se conocen aproximadamente 3000 especies con bulbos, confinadas a monocotiledóneas, en particular a Alliaceae, Amaryllidaceae, Calochortaceae, Hyacinthaceae, Iridaceae, Liliaceae y Melanthiaceae (Baranova, 2009). Muchas de las especies bulbosas han sido utilizadas desde la antigüedad por sus cualidades económicas (ornamentales, farmacéuticas, alimentarias, entre otras) y constituyen recursos genéticos de importancia para la conservación biológica dado que sus poblaciones naturales han sido depauperadas. Las estrategias más eficientes que permitan la multiplicación del material vegetal para su óptimo aprovechamiento económico, y simultáneamente evite mermar las poblaciones silvestres, resulta prioritario en especies nativas de Amaryllidaceae (Honfi \& Daviña, 2015; Honfi et al., 2017). La cualidad bulbífera, es decir, la multiplicación vegetativa mediante bulbillos hijos, es un carácter presente en varias especies de la familia, pero no es un atributo común de todas las especies (Leszczyñska-Borys \& Borys, 2001; Schiappacasse et al., 2003). La producción de plantas a partir de bulbos es un procedimiento frecuente, pero es lento (Hartmann et al., 1990). En especies de interés económico, donde se pretende incrementar la disponibilidad de plantines para la implantación de cultivos a cierta escala de producción, la multiplicación clonal de genotipos selectos, resulta un aspecto crucial a la hora de introducir materiales a planes de domesticación o mejoramiento genético.

Entre las plantas bulbosas nativas sudamericanas, las especies de los géneros Habranthus Herb., Hippeastrum Herb. y Zephyranthes Herb. (Amaryllidaceae), son particularmente utilizadas como plantas ornamentales, y en ciertos casos, como fuentes de fitoproductos, específicamente alcaloides de interés medicinal y farmacéutico (Ortiz et al., 2012, 2016; Honfi \& Daviña, 2015; Honfi et al., 2017). Hippeastrum es un género de bulbosas nativas de América tropical y subtropical, cuya mayor diversidad se encuentra al este de Brasil y en los Andes orientales de Bolivia, Perú y Argentina (Meerow \& Snijman, 1998; Meerow et al., 2000; Daviña, 2001; Dutilh, 2005; Oliveira, 2017). Hippeastrum striatum (Lam.) H. E. Moore es una especie con hojas deciduas, que presenta un escapo con dos a cuatro flores, generalmente de color naranja, a veces con tonos salmón, o más rojizos (Dutilh, 2005). Se distribuye en Brasil austral, Paraguay, Uruguay y Argentina, en nuestro país principalmente en Tucumán, Misiones, Corrientes, Entre Ríos y Buenos Aires (Arroyo-Leuenberger, 1996), en cercanías de los ríos Uruguay, Paraná y de La Plata (Arroyo-Leuenberger, 1996; Hurrell et al., 2005). Habranthus también es un género de bulbosas perennes originarias de América, presenta amplia distribución, abarcando partes del sur de Norteamérica, Centroamérica, y su mayor diversidad se encuentra en Sudamérica (Arroyo, 1990; Meerow \& Snijman, 1998; Meerow et al., 2000; Amaral Lopes \& Barbosa Cavalcanti, 2015). Habranthus brachyandrus (Baker) Sealy es una especie con hojas deciduas, presenta un escapo unifloro, con las flores de color rosado intenso y el centro más oscuro, florece principalmente en verano y se cultiva con fines ornamentales en diversos países del mundo (Jitsuno et al., 2009). Las poblaciones naturales se encuentran principalmente en Brasil, Uruguay, Paraguay y Argentina, en nuestro país con presencia importante en Chaco, Córdoba, Corrientes, Misiones, Formosa, Santa Fe y San Luis (Ravenna, 1967, 1974; Herklots, 1980; Arroyo, 1990).

Las dos especies mencionadas son adecuadas para su explotación con fines ornamentales (Leszczyñska-Borys \& Borys, 2001; Vargas et al., 2006) y para la obtención de fitoproductos (Dutilh, 2005; Vargas et al., 2006; Jitsuno et al., 2009), rasgos que las convierten en potenciales nuevos cultivos. Cuando la multiplicación natural no es suficiente, bien sea por la baja producción de semillas viables, por la poca actividad multiplicadora del bulbo madre, o simplemente porque se pretende aumentar la escala de producción, la inducción mecánica para generar nuevos bulbillos ha sido un método efectivo de bajo costo y alta eficiencia en diversas especies (Andrade-Rodríguez et al., 2015; Padilla et al., 2016). Este consiste en la realización de algún tipo de incisión en el bulbo, generalmente basales, para extirpar una porción de bulbo o dividirlo por la mitad (Daviña, 2001), de modo que las lesiones causadas estimulen la formación de bulbillos hijos (Sganzerla, 1973). El objetivo del presente trabajo es analizar la capacidad de regeneración vegetativa de bulbos de Hippeastrum striatum y Habranthus brachyandrus sometidos a inducción mecánica a través de corte longitudinal. 


\section{O. A. Rodriguez Mata et al. - Regeneración de bulbos de Amaryllidaceae}

\section{Materiales y Métodos}

El ensayo se llevó a cabo en el banco de germoplasma del programa de Estudios Florísticos y Genética Vegetal, del Instituto de Biología Subtropical (IBS-CONICET-UNaM), en un vivero construido a cielo abierto donde las plantas se cultivan en macetas, ubicado en Posadas, Misiones, Argentina. El clima local es subtropical húmedo. Las lluvias anuales rondan los $3000 \mathrm{~mm}$, con una elevada humedad ambiental (humedad relativa promedio anual: $74 \%$ ) (National Oceanic and Atmospheric Administration, 2018). Las temperaturas son templadas en invierno con un promedio de $15^{\circ} \mathrm{C}$, hay días durante los meses de junio-agosto en donde se registran temperaturas por debajo de los $10^{\circ} \mathrm{C}$. El verano tiene una temperatura promedio de $30^{\circ} \mathrm{C}$, que en ocasiones supera los $38^{\circ} \mathrm{C}$ (Servicio Meteorológico Nacional, 2018). La experiencia se realizó entre los meses de septiembre de 2017 y marzo de 2018. El cultivo se mantuvo únicamente con riego, sin agregado de fertilizantes.

\section{Material biológico}

Se utilizaron bulbos madres maduros de diferentes diámetros (Tablas 1 y 2). Se consideraron como bulbos maduros a aquellos que florecieron un año antes del ensayo (Figs. 1A; 2A). Los ejemplares de referencia se encuentran depositados en el herbario de la Universidad Nacional de Misiones (MNES). Los bulbos de $H$. striatum proceden de la accesión OR11 proveniente de ARGENTINA, Prov. Misiones: Dpto. Capital, Posadas, 23/2/2017, (S $27^{\circ} 22^{\prime} 38,9^{\prime}$ ', WO $\left.55^{\circ} 53^{\prime} 38,8^{\prime \prime}\right)$ y los de $H$. brachyandrus pertenecen a la accesión OR02, Prov. Misiones: Dpto. Capital,

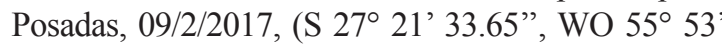
$30,58 ")$.

\section{Ensayos}

Inicialmente, los bulbos fueron despojados de sus hojas y lavados primero con agua común, y después con agua destilada. Usando un calibre de Vernier se midió el diámetro en centímetros. Se procedió a cortar cada bulbo de forma longitudinal, obteniendo dos

Tabla 1. Análisis de la capacidad regenerativa en bulbos de Hippeastrum striatum.

\begin{tabular}{|c|c|c|c|c|c|c|c|}
\hline Tratamiento & Individuo & $\begin{array}{c}\varnothing \text { Bulbo } \\
\text { Madre } \\
\mathrm{cm}\end{array}$ & $\begin{array}{c}\text { Long, Bulbo } \\
\text { Madre } \\
\text { cm }\end{array}$ & $N^{\circ}$ Bulbillos & $\begin{array}{c}\varnothing \text { bulbillo * } \\
\mathrm{cm}\end{array}$ & $\begin{array}{l}\text { Long, } \\
\text { bulbillo * } \\
\text { cm }\end{array}$ & $\mathbf{N}^{\circ}$ hojas * \\
\hline \multirow{10}{*}{$\begin{array}{l}\text { Hippeastrum striatum } \\
\text { (corte) }\end{array}$} & 1 & 5,76 & 5,60 & 20 & 1,02 & 1,26 & 1,65 \\
\hline & 2 & 3,90 & 3,50 & 16 & 1,09 & 1,21 & 1,30 \\
\hline & 3 & 3,70 & 3,40 & 13 & 1,16 & 1,24 & 1,60 \\
\hline & 4 & 5,10 & 4,80 & 19 & 1,05 & 1,15 & 1,65 \\
\hline & 5 & 4,80 & 4,30 & 17 & 1,06 & 1,19 & 1,65 \\
\hline & 6 & 3,35 & 3,00 & 15 & 1,14 & 1,22 & 1,80 \\
\hline & 7 & 3,42 & 3,10 & - & - & - & - \\
\hline & 8 & 4,75 & 4,30 & 16 & 1,11 & 1,23 & 1,40 \\
\hline & 9 & 4,20 & 3,90 & 16 & 1,12 & 1,20 & 1,40 \\
\hline & 10 & 3,62 & 3,15 & - & - & - & - \\
\hline Promedio & & 4,26 & 3,91 & 17 & 1,09 & 1,21 & 1,56 \\
\hline \multirow{5}{*}{$\begin{array}{l}\text { Hippeastrum striatum } \\
\text { (control) }\end{array}$} & 1 & 4,30 & 3,90 & 12 & 0,80 & 0,60 & 0 \\
\hline & 2 & 3,90 & 3,80 & 9 & 0,50 & 0,80 & 0 \\
\hline & 3 & 4,00 & 3,50 & 8 & 0,40 & 0,70 & 0 \\
\hline & 4 & 3,60 & 3,50 & 7 & 0,30 & 0,20 & 0 \\
\hline & 5 & 3,80 & 3,00 & 8 & 0,30 & 0,20 & 0 \\
\hline Promedio & & 3,92 & 3,54 & 9 & 0,46 & 0,50 & 0 \\
\hline
\end{tabular}

$\varnothing$ Ø: Diámetro, Long,: Longitud, $\mathrm{N}^{\circ}$ : Número, ${ }^{*}$ :Valor promedio, cm: Centímetros. 
Bol. Soc. Argent. Bot. 53 (4) 2018

Tabla 2. Análisis de la capacidad regenerativa en bulbos de Habranthus brachyandrus.

\begin{tabular}{|c|c|c|c|c|c|c|c|}
\hline Tratamiento & Individuo & $\begin{array}{c}\varnothing \text { Bulbo } \\
\text { Madre } \\
\text { cm }\end{array}$ & $\begin{array}{c}\text { Long, Bulbo } \\
\text { Madre } \\
\text { cm }\end{array}$ & $N^{\circ}$ Bulbillos & $\begin{array}{c}\varnothing \text { bulbillo * } \\
\mathrm{cm}\end{array}$ & $\begin{array}{l}\text { Long, } \\
\text { bulbillo * } \\
\text { cm }\end{array}$ & $\mathbf{N}^{\circ}$ hojas * \\
\hline \multirow{10}{*}{$\begin{array}{l}\text { Habranthus brachyandrus } \\
\text { (corte) }\end{array}$} & 1 & 2,20 & 2,80 & 21 & 0,89 & 2,08 & 1,24 \\
\hline & 2 & 2,35 & 3,10 & 20 & 0,89 & 2,31 & 1,20 \\
\hline & 3 & 3,00 & 3,40 & 16 & 0,94 & 1,73 & 1,18 \\
\hline & 4 & 2,60 & 4,60 & 17 & 0,91 & 2,00 & 1,00 \\
\hline & 5 & 2,90 & 3,20 & 17 & 0,92 & 2,18 & 1,00 \\
\hline & 6 & 2,14 & 2,70 & 23 & 0,87 & 1,97 & 1,00 \\
\hline & 7 & 2,60 & 3,00 & 16 & 0,94 & 1,88 & 1,30 \\
\hline & 8 & 3,30 & 3,50 & 16 & 0,94 & 2,00 & 1,24 \\
\hline & 9 & 2,50 & 3,00 & 19 & 0,91 & 1,90 & 1,24 \\
\hline & 10 & 3,00 & 3,80 & 16 & 0,93 & 1,95 & 1,20 \\
\hline Promedio & & 2,66 & 3,31 & 18 & 0,91 & 2,00 & 1,16 \\
\hline \multirow{5}{*}{$\begin{array}{l}\text { Habranthus brachyandrus } \\
\text { (control) }\end{array}$} & 1 & 2,80 & 3,00 & 0 & - & - & \\
\hline & 2 & 3,00 & 3,50 & 0 & - & - & \\
\hline & 3 & 2,60 & 4,30 & 0 & - & - & \\
\hline & 4 & 2,40 & 3,10 & 0 & - & - & \\
\hline & 5 & 2,75 & 3,20 & 0 & - & - & \\
\hline Promedio & & 2,71 & 3,42 & & & & \\
\hline
\end{tabular}

$\varnothing$ Ø: Diámetro, Long,: Longitud, N: Número, * :Valor promedio, cm: Centimetros.

mitades aproximadamente iguales, procurando tener la misma proporción de disco basal en cada sección (Figs. 1B; 2B). Para obtener mayor precisión en los cortes se utilizó un cuchillo de hoja de cerámica. Los bulbos seccionados de $H$. striatum se plantaron en macetas de $7,0 \mathrm{~cm}$ de diámetro por $8,5 \mathrm{~cm}$ de altura. A los 45 días se fotografiaron los bulbos con brotes (Fig. 1C). Los bulbos cortados de H. brachyandrus se plantaron en bandejas rectangulares de $20 \mathrm{~cm}$ de largo por $15 \mathrm{~cm}$ de ancho, a razón de 3 bulbos por bandeja, emparejando las mitades de un mismo individuo. A los 45 días se fotografiaron los bulbos con brotes (Fig. 2C) y a los 3 meses se trasplantaron las mitades de bulbo con brotes a macetas más grandes de $12 \mathrm{~cm}$ de diámetro por $10 \mathrm{~cm}$ de altura. Se empleó como sustrato una mezcla de proporción 3:2:1 de tierra, arena y sustrato profesional compuesto de turba de musgo Sphagnum de fibras medias, compost de corteza, cal calcita, cal dolomita y agentes humectantes. El riego fue suministrado cada 48 hs durante las primeras dos semanas después de plantadas las mitades. Luego de los 15 días se regaron 2 veces por semana hasta completar el primer mes. A los 30 días el riego se realizó una vez por semana. Se regó más frecuentemente según aumentaban las temperaturas con la cercanía del verano. Como tratamiento control se sembraron bulbos maduros enteros, en las mismas condiciones que los sometidos a corte.

\section{Diseño experimental}

Se estableció un diseño completamente al azar con 10 repeticiones. La unidad experimental estuvo constituida por un bulbo, seccionado en dos partes. El monitoreo se realizó semanalmente. A los 6 meses (Figs. 1D; 2D) se hizo la evaluación final de las variables de estudio (Figs. 1E; 2E).

\section{Variables de estudio}

Las variables evaluadas fueron: número de bulbillos generados por cada bulbo madre, longitud 


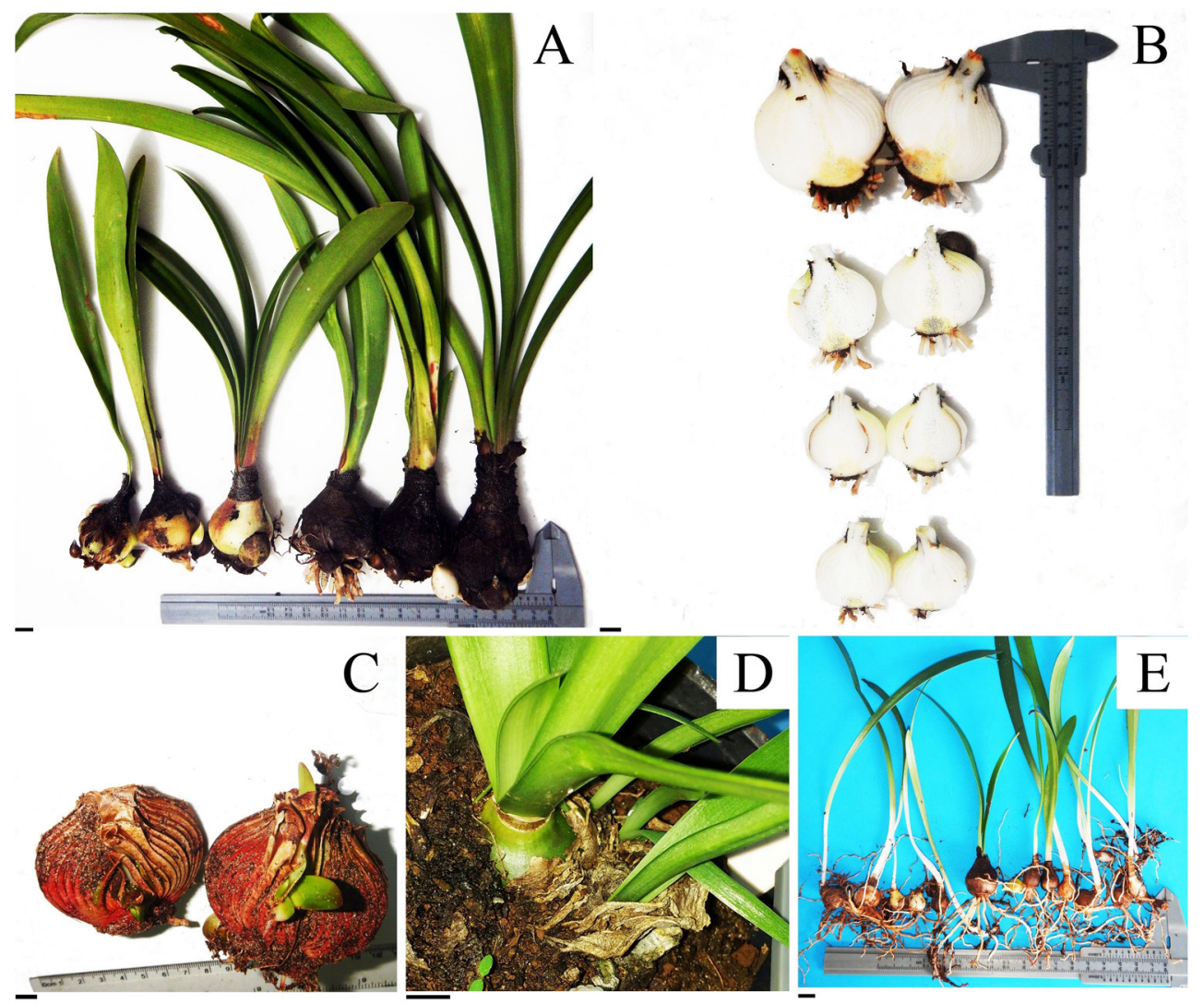

Fig. 1. Hippeastrum striatum. A: Bulbos madre. B: Bulbos cortados C: Brotes de los bulbos cortados a los 45 días posteriores al corte. D: Bulbillos hijos de bulbos cortados a los seis meses. E: Detalle de los nuevos clones a los 6 meses de ensayo. Escalas: A-C: $2 \mathrm{~cm}$; D: $1 \mathrm{~cm}$; E: $5 \mathrm{~cm}$.

y diámetro promedio de bulbillos y número de hojas de los bulbillos generados. Los datos obtenidos fueron examinados mediante análisis de varianza, prueba de comparación de medias y regresión simple, usando STATGRAPHICS Centurión XV.II.

\section{Resultados y Discusión}

La inducción bulbífera mecánica a través del corte longitudinal en el bulbo madre tuvo un efecto altamente significativo $(p \leq 0.05)$ en la producción de nuevos bulbillos (Tablas 1-3). Hippeastrum striatum y Habranthus brachyandrus producen naturalmente bulbillos hijos. Los bulbos de $H$. striatum son subglobosos, prolíficos, de $4-8 \mathrm{~cm}$ de diámetro, y presentan colores en tonos castañonegruzcos (Hurrell et al., 2005). Los bulbos de $H$. brachyandrus también son subglobosos, apenas deprimidos, de 2-3,3 $\mathrm{cm}$ de diámetro, frecuentemente alargados, y presentan colores en tonos castaños oscuros a negros (Hurrell et al., 2005). En los ensayos con, H. brachyandrus, las unidades de estudios resistieron el corte en el 100\% de los casos. En H. striatum las unidades de estudio resistieron el corte en el $80 \%$ de los casos: dos de los diez bulbos cortados murieron durante el desarrollo del ensayo y fueron los de menor tamaño.

Número de bulbillos producidos por cada bulbo madre

En ambas especies el número promedio de bulbillos hijos fue mayor respecto de los testigos (Tablas 1 y 2). En los bulbos cortados de $H$. striatum, el rango de producción fue de 13 a 20 nuevos bulbillos por cada bulbo madre, que representa en promedio $87,5 \%$ más producción respecto del control, cuyo rango de producción fue 
Bol. Soc. Argent. Bot. 53 (4) 2018
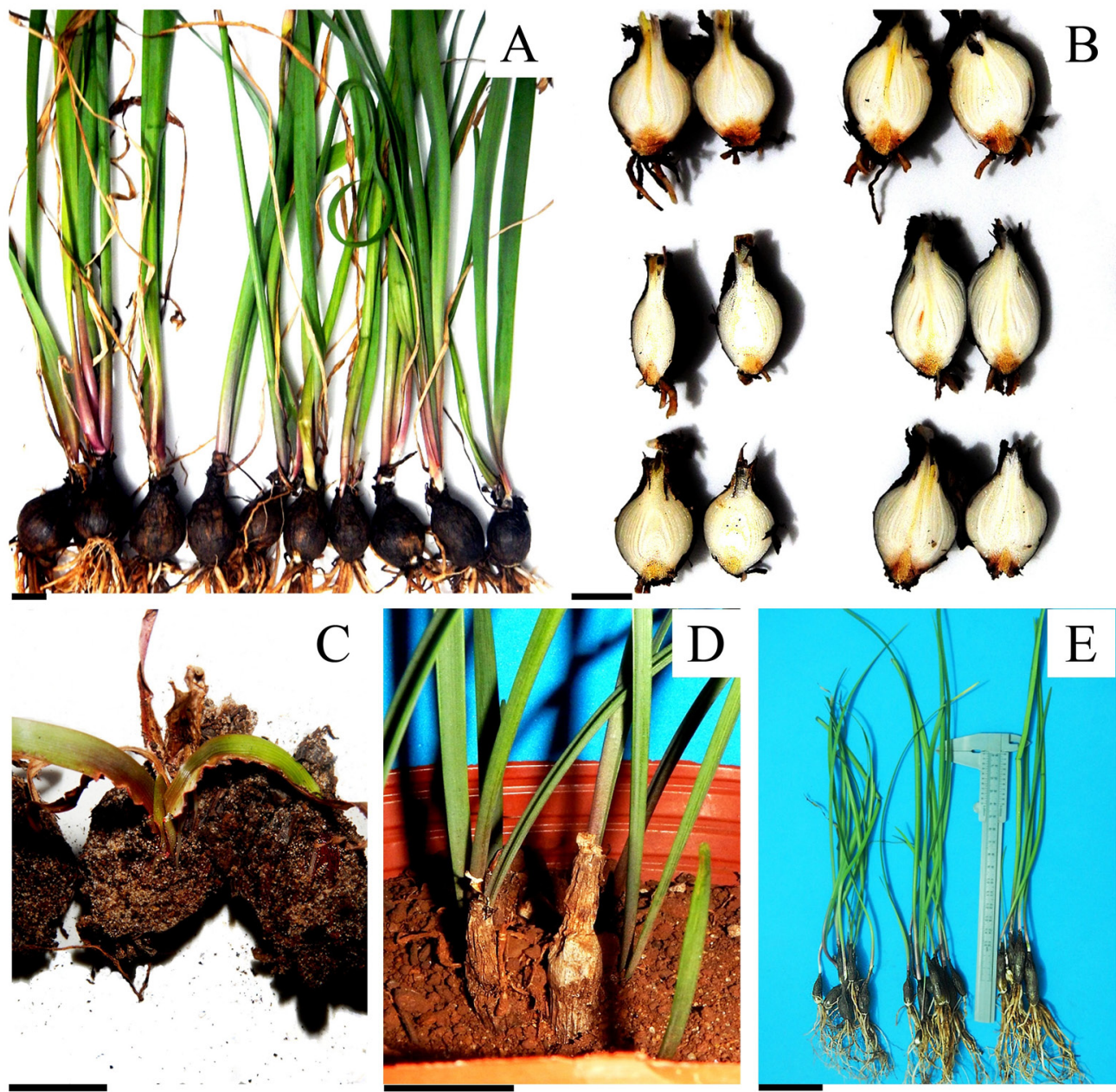

Fig. 2. Habranthus brachyandrus. A: Bulbos madre. B: Bulbos cortados C: Brotes de los bulbos cortados a los 45 días posteriores al corte. D: Bulbillos hijos de bulbos cortados a los seis meses. E: Detalle de los nuevos clones a los 6 meses de ensayo. Escalas: A-C: $2 \mathrm{~cm}$; D: $1 \mathrm{~cm} ; \mathbf{E}: 5 \mathrm{~cm}$.

Tabla 3. Resumen estadístico de bulbillos producidos en Hippeastrum striatum.

\begin{tabular}{|lcc|}
\hline \multicolumn{1}{c}{ Estadístico } & $\mathbf{N}^{\circ}$ bulbillos corte & $\mathbf{N}^{\circ}$ bulbillos control \\
\hline Promedio & 16,5 & 8,8 \\
Mediana & 16 & 8 \\
Desviación Estándar & 2,20389 & 1,92354 \\
Coeficiente de Variación & $13,36 \%$ & $21,86 \%$ \\
Error Estándar & 0,779194 & 0,860233 \\
Mínimo & 13 & 7 \\
Máximo & 20 & 12 \\
Rango & 7 & 5 \\
Sesgo Estandarizado & 0,246559 & 1,38526 \\
Curtosis Estandarizada & 0,0615308 & 1,19027 \\
Valor-P Comparación de medias & & 0,00004992 \\
\hline
\end{tabular}


de 7 a 12 nuevos bulbillos por bulbo madre (Tabla 1). En los bulbos cortados de H. brachyandrus el rango de producción fue de 16 a 23 nuevos bulbillos por bulbo madre (Tabla 2). En el tratamiento control de $H$. brachyandrus no hubo producción de nuevos bulbillos. En general, la formación de nuevos bulbillos fue siempre menor en los bulbos que fueron plantados enteros (tratamiento control). Los resultados indican que los cortes realizados estimularon el desarrollo vegetativo y una mayor producción de bulbillos. De modo similar, Ephrath et al. (2001), señalan que en Hippeastrum hybridum, var. Red Lion, se obtuvo mayor número de bulbillos al aumentar la cantidad de secciones en que se dividió el bulbo madre. También, la cantidad de bulbillos hijos puede estar relacionada con el tamaño del bulbo maduro de la especie, así, por ejemplo, en especies de Hymenocallis Salisb., se obtuvieron de 21 a 40 bulbillos/ bulbo, durante la propagación clonal estimulada por cortes (Leszczyñska et al., 2005). En nuestro caso, en ambas especies la cantidad de bulbillos clonales fue mayor en los ensayos con cortes, con una relación estadísticamente significativa entre el diámetro del bulbo madre y la cantidad de bulbillos producidos. En $H$. striatum la correlación es positiva (Fig. 3A), y existe además, una relación significativa entre el diámetro del bulbo madre y su longitud (Fig. 3B). En cambio, en $H$. brachyandrus la correlación entre el diámetro del bulbo madre y la cantidad de bulbillos producidos es negativa (Fig. 4A), y no existe una relación significativa entre el diámetro del bulbo madre y su longitud (Fig. 4B), posiblemente debido a que el aumento del tamaño de los bulbos de esta especie ocurre en sentido longitudinal más que en sentido radial, razón por la cual son más o menos alargados independientemente del diámetro.

Los resultados sugieren para ambas especies que la inducción bulbífera a través del corte estimuló el crecimiento de las yemas basales de las catáfilas, y la emergencia de los bulbillos en cada sección. El daño provocado al cortar el bulbo y la inhibición de la dominancia de la yema principal serían los responsables de estimular la activación de las yemas basales. Sganzerla (1973), señala que diferentes tipos de cortes en el bulbo, incluso extirpar una porción de bulbo, o seccionarlo a la mitad, induce la activación de las yemas a través de un mecanismo hormonal en algunas bulbosas con flores. En el tratamiento control, la yema principal permaneció intacta y se produjo menor número de
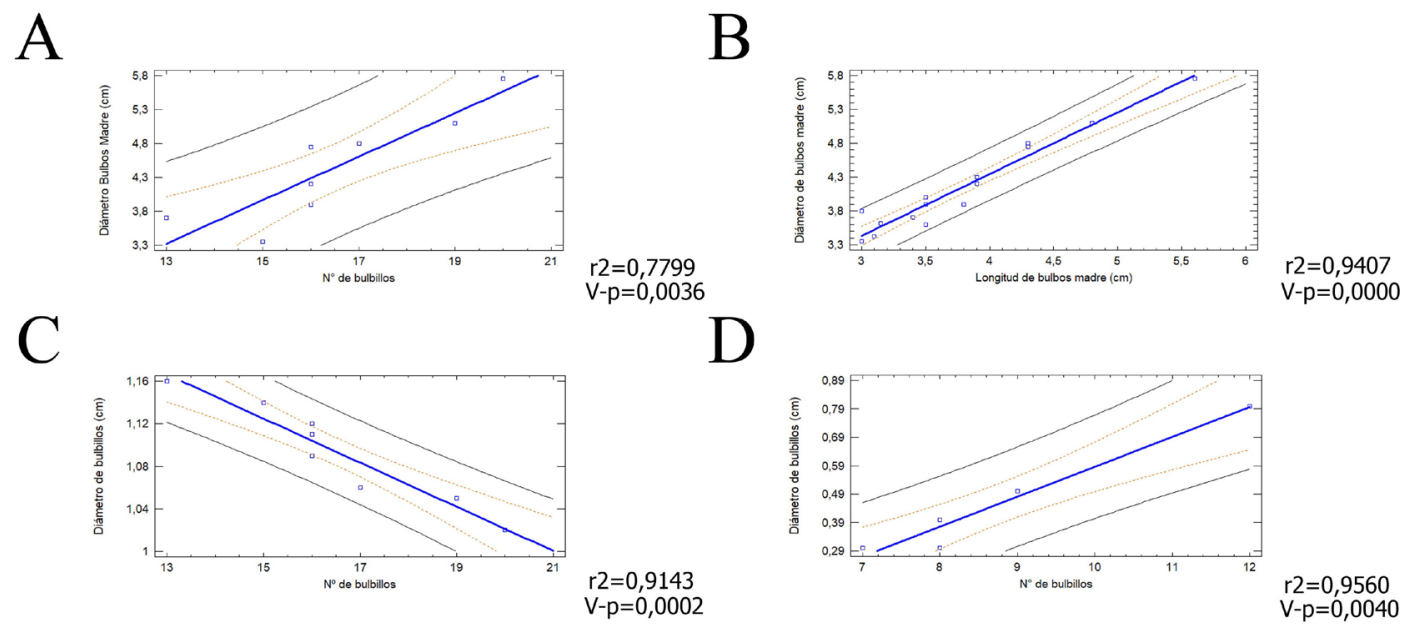

Fig. 3. Hippeastrum striatum. A: Regresión lineal del diámetro del bulbo madre vs número de bulbillos producidos (tratamiento corte). B: Regresión lineal del diámetro vs longitud de los bulbos madre. C: Regresión lineal del número de bulbillos producidos vs diámetros (tratamiento corte). D: Regresión lineal del número de bulbillos producidos vs diámetros (tratamiento control). Las líneas externas representan los límites de predicción, las líneas punteadas representan los límites de confianza, la línea central es la pendiente del modelo ajustado. El nivel de significancia de los límites de confianza y de predicción es del $95 \%$. 


\section{Bol. Soc. Argent. Bot. 53 (4) 2018}

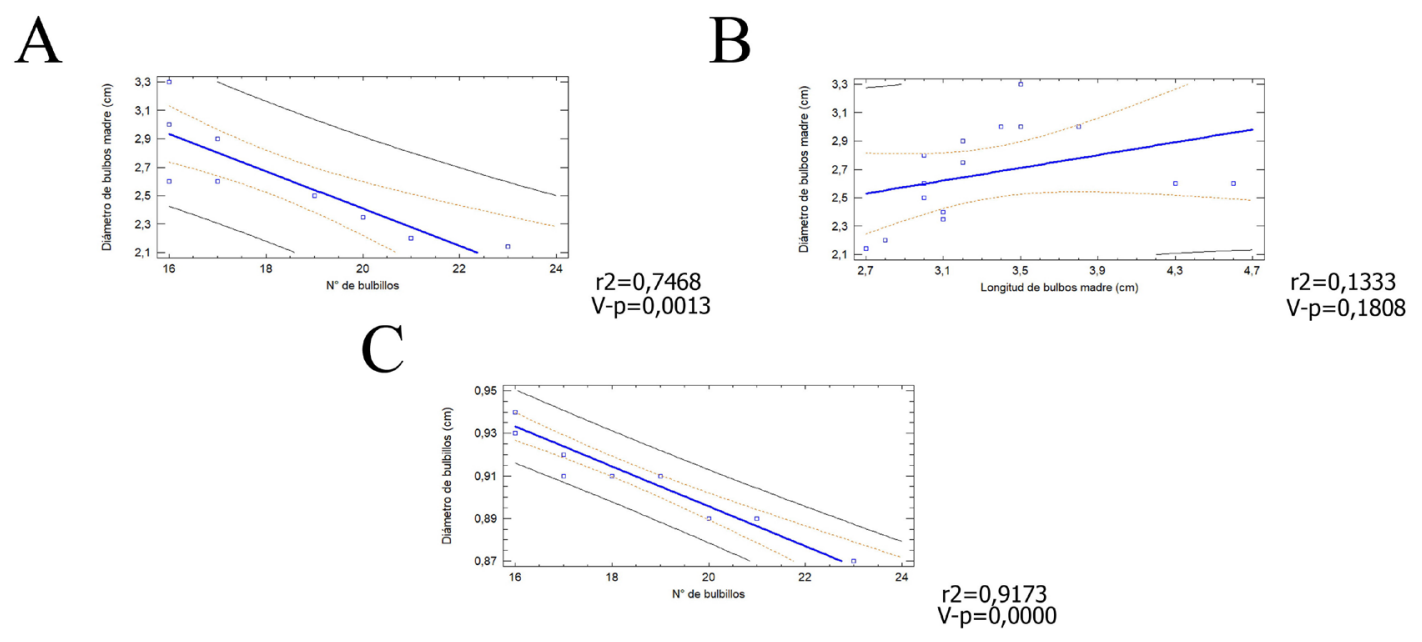

Fig. 4. Habranthus brachyandrus. A: Regresión lineal del diámetro del bulbo madre vs número de bulbillos producidos (tratamiento corte). B: Regresión lineal del diámetro vs longitud de los bulbos madre. C: Regresión lineal del número de bulbillos producidos vs diámetros (tratamiento corte). Las líneas externas representan los límites de predicción, las líneas punteadas representan los límites de confianza, la línea central es la pendiente del modelo ajustado. El nivel de significancia de los límites de confianza y de predicción es del $95 \%$.

nuevos bulbillos en $H$. striatum y no se produjeron en H. brachyandrus. Azcón-Bieto \& Talón (2008), indican que se ha comprobado que la yema apical puede inhibir el transporte polar de la auxina endógena hacia las yemas laterales, transporte que sería indispensable para su crecimiento, dado que estos reguladores estimulan el crecimiento de la planta madre e inhiben el crecimiento de las yemas laterales. Schiappacasse et al. (2003), observaron que los bulbos no divididos de Phycella australis (Ravenna) aumentaron de tamaño, pero fueron prácticamente incapaces de originar bulbillos. Sin embargo, la eliminación de la yema principal en bulbos de Ornithogalum L., estimuló una mayor producción de bulbillos (Kariuki, 2008). Del mismo modo, en los bulbos control de H. striatum y $H$. brachyandrus, cuya yema principal del bulbo se mantuvo intacta, es posible que la dominancia apical haya inhibido el crecimiento de las yemas internas presentes entre los catáfilos.

\section{Características de los bulbillos}

En los bulbos cortados de H. striatum los nuevos bulbillos producidos alcanzaron diámetros que variaron entre los 1,02 y $1,16 \mathrm{~cm}(\mathrm{x}=1,09 \mathrm{~cm})$ al cabo de 6 meses de desarrollo (Tabla 1). Por su parte, en los bulbos cortados de H. brachyandrus, los nuevos bulbillos alcanzaron diámetros que variaron entre los 0,87 y $0,94 \mathrm{~cm}(\mathrm{x}=0,91 \mathrm{~cm})$ en el mismo tiempo de desarrollo (Tabla 2). Las diferencias entre ambas están relacionadas al tamaño promedio habitual de los bulbos maduros propios de cada especie. En ambas especies se observó que a mayor número de bulbillos generados por bulbo madre, cada uno de éstos, presentó menor diámetro promedio, indicando una correlación negativa $(p \leq 0.05)$ (Figs. 3C; 4C). Hanks (1993) menciona que existe una relación inversa entre el tamaño y el número de los bulbillos producidos, en especies del género Narcissus L. También en este mismo sentido, en Hippeastrum hybridum, var. Red Lion, se observó que, al desarrollarse menor número de bulbillos, hubo mayor cantidad de reservas disponibles para que pudieran lograr un mayor crecimiento (Ephrath et al., 2001). En nuestro ensayo, se observó que los bulbos enteros de $H$. striatum produjeron bulbillos de menor diámetro promedio respecto de los bulbillos formados en los bulbos cortados (Tabla 1), posiblemente debido a que, en bulbos enteros se expresó el efecto dominante de la yema principal del bulbo madre. Aun así, en este caso (control), la correlación entre el número de bulbillos y el diámetro de los mismos es positiva $(p \leq 0.05)$ (Fig. 3D), a diferencia de 


\section{O. A. Rodriguez Mata et al. - Regeneración de bulbos de Amaryllidaceae}

la correlación negativa obtenida en los bulbillos del tratamiento corte (Fig. 3C). Estos resultados sugieren que a mayor cantidad de nuevos bulbillos menor cantidad de reservas disponibles para que éstos puedan robustecerse.

En $H$. striatum se obtuvieron bulbillos cuyas longitudes variaron entre los $1,15 \mathrm{~cm}$ y $1,26 \mathrm{~cm}$, con promedio de 1,21 cm (Tabla 1). En H. brachyandrus, se obtuvieron longitudes que variaron entre los 1,73 $\mathrm{cm}$ y $2,31 \mathrm{~cm}$, con promedio de $2,00 \mathrm{~cm}$ (Tabla 2). En ambas especies, la mayor longitud se presentó en bulbillos de bulbos cortados, respecto al control. Coincidiendo con Padilla et al. (2016), quienes observaron en el género Zephyranthes que los bulbillos de menor longitud correspondieron a los bulbos plantados enteros. Al igual que en la variable anterior, el efecto dominante de la yema principal en los bulbos control, pudieron haber retrasado el crecimiento de los nuevos individuos.

En ambas especies se produjeron hojas solamente en los bulbillos de los bulbos cortados. La cantidad varió entre 0-5 hojas en $H$. striatum con promedio de 1,56 hojas/bulbillo (Tabla 1) y entre 0-3 en $H$. brachyandrus con promedio de 1,16 hojas/bulbillo (Tabla 2). En los ensayos control, solo H. striatum produjo nuevos bulbillos y estos no mostraron desarrollo de hojas.

\section{Conclusiones}

La inducción bulbífera mecánica a través de cortes, aplicados en bulbos de $H$. striatum y $H$. brachyandrus estimulan el proceso de activación de las yemas basales del bulbo madre, provocando la aparición de nuevos bulbillos clonales, y, representa una alternativa para la propagación vegetativa rápida y poco onerosa de estas especies promisorias. Es una herramienta valiosa a tener en cuenta a la hora de implementar planes de conservación, mejoramiento, aprovechamiento comercial con fines ornamentales, obtención de fitoproductos $\mathrm{u}$ otros usos, que requieran multiplicación a gran escala.

\section{Bibliografía}

AMARALLOPES,A.C.\&T.BARBOSACAVALCANTI. 2015. Habranthus (Amaryllidaceae) do Brasil. Rodriguésia 66: 203-220.
ANDRADE-RODRÍGUEZ, M., D. GUILLÉN, O. VILLEGAS, J. J. AYALA, V. LÓPEZ \& J. VARGAS. 2015. Forma de corte del bulbo para la propagación de mancuernilla (Hippeastrum hybridum Hort.). Revista Chapingo Ser. Hort. 21: 57-69.

ARROYO, S. 1990. Habranthus (Amaryllidaceae) en Argentina y Uruguay. Parodiana 6: 11-30.

ARROYO-LEUENBERGER, S. C. 1996. Amaryllidaceae. En: ZULOAGA F. \& O. MORRONE (eds.), Catálogo de las Plantas Vasculares de la República Argentina I. Monogr. Syst. Bot. Missouri Bot. Gard. 60: 90-100.

AZCÓN-BIETO, J. \& M. TALÓN. 2008. Fundamentos de Fisiología Vegetal. MC Graw Hill Interamericana. Madrid.

BARANOVA, M. B. 2009. Bulbs, bulblet and bulbils. In: BATYGINA B. (ed.), Embryology of Flowering Plants, pp. 239-244. Science Publishers, New Humpshire.

BATYGINA, T. B. 2009. Reproduction, propagation and renewal. In: BATYGINA B. (ed.), Embryology of Flowering Plants, pp. 15-19. Science Publishers, New Humpshire.

DAVIÑA, J. R. 2001. Estudios citogenéticos en algunos géneros argentinos de Amaryllidaceae. Universidad Nacional de Córdoba. Tesis Doctoral, 184 pp.

DE ABATE, J. 1999. Biología Aplicada. 3a. ed. EUNED, San José, Costa Rica.

DUTILH, J. H. A. 2005. Ornamental bulbous plants of Brazil. Acta Hort. 683: 37-42.

EPHRATH, J. E., J. BEN-ASHER, F. BARUCHIN, C. ALEKPEROV, E. DAYAN \& M. SILBERBUSH. 2001. Various cutting methods for the propagation of Hippeastrum bulbs. Biotronics 30: 75-83.

HANKS, G. 1993. Narcissus. In: DE HERTOGH, A. \& M. LE NARD (Eds.). The physiology of flower bulbs, pp. 463-558, Elsevier. Amsterdam.

HARTMANN, H. D. \& KESTER, D. E. 1975. Propagación de plantas: principios y prácticas. 4a. ed. Compañía Editorial Continental, México.

HARTMANN, H. T., D. E KESTER, F. T DAVIES \& R. GENEVE. 1990. Plant propagation: principles and practices. Englewood Cliffs, Prentice-Hall. New Jersey.

HERKLOTS, G.A. C. 1980. Wind flowers: part 2. Habranthus. Plantsman 2: 90-99.

HONFI, A. I. \& J. R. DAVIÑA. 2015. Flora de Interés Forrajero y Ornamental de Campo San Juan. En: BAUNI V. \& M. A. HOMBERG (eds.), Reserva Natural Campo San Juan, pp. 53 - 68. Editorial Fundación de Historia Natural Félix de Azara, Ciudad Autónoma de Buenos Aires.

HONFI, A. I., A. D. BOLZÁN \& J. R. DAVIÑA. 2017. Dimensión Cromosómica. Ci. \& Invest. AAPC. 67: $5-24$.

HURRELL, J. A., D. H. BAZZANO \& G. DELUCCHI. 2005. Biota Rioplatense X Monocotiledóneas Herbáceas Nativas y Exóticas. 1'. Ed. LOLA. Buenos Aires. 
KARIUKI, W. 2008. Rapid multiplication of Ornithogalum saundersiae Bak. through bulblet production in vivo. Acta Hort. 766: 135-141.

LESZCZYÑSKA-BORYS, H. \& W. M. BORYS. 2001. Plantas bulbosas para flor de corte, macetas, jardines y parques. SIZA-CONACYT-UPAEPFUPPUE. México.

LESZCZYÑSKA, H., W. M. BORYS \& S. J. GALVÁN. 2005. Cloning of the Hymenocallis. Acta Hort. 673: 691-697.

JITSUNO, M., A. YOKOSUKA, H. SAKAGAMI \& Y. MIMAKI. 2009. Chemical constituents of the bulbs of Habranthus brachyandrus and their Cytotoxic Activities. Chem. Pharm. Bull. 57: 1153-1157.

MEEROW, A. W. \& SNIJMAN D. A. 1998. The families and genera of vascular plants. Vol. 3, Monocotyledons: Lilianae (except Orchidaceae), 83-110. Royal Botanic Gardens edition. Berlin: Springer-Verlag.

MEEROW, A. W, M. W. CHASE, C. L. GUY, Q. B. LI, D. SNIJMAN \& S. Y. YANG. 2000. Phylogeny of Amaryllidaceae: molecules and morphology. In: WILSON K. \& D. MORRISON (eds.), Monocots, systematics and evolution, pp. 368-382. CSIRO, Sidney.

NATIONAL OCEANIC AND ATMOSPHERIC ADMINISTRATION. Posadas Aero Climate Normals 1961-1990. Disponible en: ftp://ftp.atdd. noaa.gov/pub/GCOS/WMO-Normals/TABLES/ REGIII/AG/87178.TXT [Acceso: 16 marzo 2018].

OLIVEIRA, R., J. D. URDAMPILLETA \& J. H. A. DUTILH. 2017. A new Hippeastrum (Amaryllidaceae) species from Brazil. Phytotaxa 307: 147-152.

ORTIZ, J. E., N. B. PIGNi, S. A., ANDUJAR, G. ROITMAN, F. D. SUVIRE, R. D. ENRIZ, A. TAPIA, J. BASTIDA \& G. E. FERESIN. 2016. Alkaloids from Hippeastrum argentinum and their Cholinesterase-Inhibitory Activities: An in Vitro and in Silico Study. J. Nat. Prod. 79: 1241-1248.
ORTIZ, J. E., S. BERKOV, N. B. PIGNI, C. THEODULOZ, G. ROITMAN, A. TAPIA, J. BASTIDA \& G. E. FERESIN. 2012. Wild Argentinian Amaryllidaceae, a new renewable source of the Acetylcholinesterase inhibitor Galanthamine and other alkaloids. Molecules, 17: 13473-13482.

PADILLA, A., M. ANDRADE, I. TEJACAL, O. VILLEGAS \& D. GUILLÉN. 2016. Vegetative and sexual propagation of mayito (Zephyranthes fosteri Traub and Zephyranthes lindleyana Herb.) (Amaryllidaceae). Acta Agrícola y Pecuaria 2: 3442.

RAVEN, P.H., EVERT \& R.F., EICHHORN, S.E. 2007. Biología vegetal $7 \mathrm{ma}$. ed. Rio de Janeiro, Guanabara Koogan.

RAVENNA, P. F. 1967. Sillent Hill Contribuições ao estudo das Amaryllidaceae da América do Sul. Sellowia 19: 25-36.

RAVENNA, P. F. 1974. Contributions to South American Amaryllidaceae VI. Pl. Life 30: 29-79.

SCHIAPPACASSE, F., P. PEÑAILILLO \& P. YAÑEZ. 2003. Propagación de bulbosas chilenas ornamentales. Editorial Universidad de Talca. Chile.

SERVICIO METEOROLÓGICO NACIONAL. Valores Medios de Temperatura y PrecipitaciónMisiones: Posadas. Consultado. Disponible en: https://www.smn.gob.ar/caracterizaci $\% \mathrm{C} 3 \% \mathrm{~B} 3 \mathrm{n}-$ estad\%C3\%ADsticas-de-largo-plazo [Acceso: 16 marzo 2018].

SGANZERLA, M. 1973. Flores de bulbo, cómo cultivarlas. 3a Ed. Vecchi. Barcelona, España.

VARGAS, T.E., M. OROPEZA \& E. DE GARCÍA. 2006. Propagación in vitro de Hippeastrum sp. Agron. Trop. 56: 621-626.

Recibido el 13 de agosto de 2018, aceptado el 23 de octubre de 2018. Editor: Federico Mollard. 